\title{
Nontuberculous mycobacteria in respiratory samples from patients with pulmonary tuberculosis in the state of Rondônia, Brazil
}

\author{
Cleoni Alves Mendes de Lima ${ }^{1,2 /+}$, Harrison Magdinier Gomes ${ }^{3}$, \\ Maraníbia Aparecida Cardoso Oelemann ${ }^{3}$, Jesus Pais Ramos ${ }^{4}$, Paulo Cezar Caldas ${ }^{4}$, \\ Carlos Eduardo Dias Campos ${ }^{4}$, Márcia Aparecida da Silva Pereira ${ }^{3}$, Fátima Fandinho Onofre Montes ${ }^{4}$, \\ Maria do Socorro Calixto de Oliveira', Philip Noel Suffys ${ }^{3}$, Maria Manuela da Fonseca Moura ${ }^{1}$
}

\begin{abstract}
${ }^{1}$ Centro Interdepartamental de Biologia Experimental e Biotecnologia, Universidade Federal de Rondônia, Porto Velho, RO, Brasil ${ }^{2}$ Laboratório Central de Saúde Pública de Rondônia, Porto Velho, RO, Brasil ${ }^{3}$ Laboratório de Biologia Molecular Aplicada a Micobactérias, Instituto Oswaldo Cruz ${ }^{4}$ Centro de Referência Professor Hélio Fraga, Escola Nacional de Saúde Pública-Fiocruz, Rio de Janeiro, RJ, Brasil
\end{abstract}

The main cause of pulmonary tuberculosis (TB) is infection with Mycobacterium tuberculosis (MTB). We aimed to evaluate the contribution of nontuberculous mycobacteria (NTM) to pulmonary disease in patients from the state of Rondônia using respiratory samples and epidemiological data from TB cases. Mycobacterium isolates were identified using a combination of conventional tests, polymerase chain reaction-based restriction enzyme analysis of hsp65 gene and hsp65 gene sequencing. Among the 1,812 cases suspected of having pulmonary TB, 444 yielded bacterial cultures, including 369 cases positive for MTB and 75 cases positive for NTM. Within the latter group, 14 species were identified as Mycobacterium abscessus, Mycobacterium avium, Mycobacterium fortuitum, Mycobacterium intracellulare, Mycobacterium gilvum, Mycobacterium gordonae, Mycobacterium asiaticum, Mycobacterium tusciae, Mycobacterium porcinum, Mycobacterium novocastrense, Mycobacterium simiae, Mycobacterium szulgai, Mycobacterium phlei and Mycobacterium holsaticum and 13 isolates could not be identified at the species level. The majority of NTM cases were observed in Porto Velho and the relative frequency of NTM compared with MTB was highest in Ji-Paraná. In approximately half of the TB subjects with NTM, a second sample containing NTM was obtained, confirming this as the disease-causing agent. The most frequently observed NTM species were M. abscessus and $\mathrm{M}$. avium and because the former species is resistant to many antibiotics and displays unsatisfactory cure rates, the implementation of rapid identification of mycobacterium species is of considerable importance.

Key words: pulmonary tuberculosis - nontuberculous mycobacteria - PRA - Rondônia - Brazil

Tuberculosis (TB) is a major health problem in many countries and is primarily caused by organisms of the Mycobacterium tuberculosis complex (MTBC). However, the contribution of nontuberculous Mycobacterium (NTM) species to disease has also been reported and appears to depend on both environmental and host factors, leading to a variety of clinical symptoms (Griffith et al. 2007). Despite being isolated frequently from environmental and human samples, NTM species were recognised as a cause of human disease only at the end of the fifties (Brachaman \& Abrutyn 2009). A re-evaluation of NTM species as disease-causing agents occurred due to the association of NTM infection with acquired immune deficiency syndrome (AIDS) and the recognition of NTM as the causative agent of lung disease in human immunodeficiency virus (HIV)-negative cases (Griffith et al. 2007). The clinical features of lung disease caused by NTM are often very similar to those of TB (Pedro et al. 2008). At present, over 150 different

doi: $10.1590 / 0074-0276108042013010$

+ Corresponding author: cleoniml@yahoo.com.br

Accepted 14 August 2012

Received 26 November 2012 species of NTM have been described and NTM infections are emerging in scenarios not previously recognised, including in Brazil, with new clinical manifestations (Griffith et al. 2007).

Outbreaks of NTM infections are increasing worldwide (Wallace et al. 1998) and also in Brazil. Initially this was due to the HIV/AIDS pandemic, but now is apparently associated with surgery and cosmetic procedures due to the use of improperly sterilised equipment (MS 2008).

Rapid detection and identification of mycobacterium at the species level is important for the accurate selection and administration of antibiotics for infection and transmission control, particularly in immunosuppressed patients (Katoch 2004). The improvement in the techniques designed for mycobacterium identification in clinical laboratories has resulted in faster and more accurate identification of NTM on a larger scale and even directly in clinical samples (Griffith et al. 2007). Traditionally, the diagnosis of TB and NTM has depended on bacilloscopy and culturing in specific medium, but culture-based identification methods using biochemical tests are slow and inadequate to differentiate the growing list of mycobacterium species (Dai et al. 2011).

In Brazil, in the state of Rondônia (RO), mycobacterium culturing is not performed as a routine procedure for the diagnosis of TB in all symptomatic patients with 
negative sputum smears and there are no records of studies on NTM in RO. Therefore, our main objective was to study the frequency and diversity of NTM species present in pulmonary samples of patients with lung disease and evaluate their geographical distribution within the areas of occurrence in RO.

\section{SUBJECTS, MATERIALS AND METHODS}

Patient isolates - We performed a retrospective study using the laboratory data collected during routine processing of sputum samples obtained from individuals suspected of having pulmonary TB who were sent to the Central Laboratory of Public Health in RO (LACEN). Our study included 1,812 cultures obtained from 1,301 individuals who had at least one culture positive for mycobacterium and were either under investigation or being treated for disease between January 2008-December 2010. The laboratory data included the number and type of specimens collected, site of isolation, number of positive cultures obtained from the same case, identification of bacterial species as determined by conventional identification methods and HIV status of the patient. This study was approved by the Ethical Committee of the Federal University of Rondônia (protocol 19/2007).

Sample processing, culturing and mycobacterium identification - The samples were processed according to standard isolation techniques following the rules, regulations and recommendations described in the Manual for Tuberculosis Bacteriology of the Ministry of Health (MS 2005).

Distinction between MTBC and NTM species was determined by macroscopic and microscopic observation of cultures in Löwenstein-Jensen (LJ), the presence of cord factor and testing bacterial growth inhibition in medium containing $0.5 \mathrm{mg} / \mathrm{mL}$ para-nitrobenzoicacid (MS 2005). After characterisation of mycobacteria at the LACEN, the samples were sent to Reference Centre Professor Hélio Fraga at the National Reference Centre for Tuberculosis (Curicica, state of Rio de Janeiro) for species identification by polymerase chain reaction (PCR)-based restriction enzyme analysis (PRA) of a 441 bp fragment of the $h s p 65$ gene (Telenti et al. 1993). In the samples with ambiguous PRA patterns, sequencing of the $h s p 65$ PCR products was performed as described previously (McNabb et al. 2004).

The prevalence of NTM was defined as the number of individuals who presented at least one sample with a positive culture, while the microbiological criterion for pulmonary NTM as recommended by the American Thoracic Society (ATS) is obtaining positive cultures from at least two different samples of expectorated sputum.

\section{RESULTS}

We analysed 1,812 respiratory secretion samples obtained from 1,301 individuals who were either suspected of having or were being treated for pulmonary TB. Among them, 444 (24.5\%) yielded Mycobacterium growth in LJ, $369(83.1 \%)$ were identified as belonging to the MTBC and 75 (16.9\%) were NTM.
Among the 45 individuals who yielded the 75 NTM isolates, we received more than one sample from 24 cases and only two individuals did not have consistent positive culture-results (the 1st sample was positive and the 2 nd was negative). The other 19 cases $(46.7 \%)$ had positive cultures from either two $(n=12)$, three $(n=4)$, four $(\mathrm{n}=2)$ or five $(\mathrm{n}=1)$ consecutive samples and, according to the microbiological criteria defined by the ATS, were suffering from lung disease caused by NTM.

Among the 45 NTM-positive subjects, 29 (64.5\%) were male and the subjects' age ranged from 12-78 years, with an average age of 50 years. Among the cases with TB caused by MTBC, 232 (72\%) were male, with a median age of 36.7 years.

The RO comprises 52 municipalities and 24 of these municipalities sent out samples to perform Mycobacterium culture for a routine test as part of the National Tuberculosis Control Program. Of these, eight (33.3\%) resulted in positive cultures for NTM (Table I).

Overall, the most frequent species throughout the state was identified as Mycobacterium abscessus $(\mathrm{n}=24$, $32 \%$ ), followed by Mycobacterium avium ( $\mathrm{n}=13,17.3 \%$ ) and Mycobacterium fortuitum $(\mathrm{n}=9,12 \%)$. Thirteen NTM isolates (21\%) remained unidentified because they could not be submitted for molecular identification.

In total, we identified 14 species of NTM, 12 isolated in Porto Velho (85.7\%), six in Ji-Paraná (42.8\%), two in Ariquemes (14.3\%) and three in other districts (21.4\%). We also evaluated regional mycobacterium species frequencies, as demonstrated in Table II.

Sequencing was performed in two cases in which PRA-hsp6 could not identify NTM at the species level. One isolate showed $99.75 \%$ identity with $M$. avium ATCC 25291 (GQ153289.1), while the other had 99.75\% similarity with Mycobacterium boenickei CIP 107829 (107829). The isolate identified by PRA-hsp65 as Mycobacterium novocastrense was confirmed by partial sequencing of the hsp65 gene (Table III).

\section{TABLE I}

Distribution of cases of Mycobacterium tuberculosis and nontuberculous Mycobacterium (NTM) in municipalities with higher occurrence in the state of Rondônia between 2008-2010

\begin{tabular}{lcccc}
\hline & \multicolumn{4}{c}{ Culture results } \\
\cline { 2 - 3 } & \multicolumn{2}{c}{ Positive } & & \multicolumn{2}{c}{ Negative } \\
\cline { 2 - 4 } \cline { 5 - 6 } Municipalities & $\begin{array}{c}\text { Tuberculosis } \\
\mathrm{n}(\%)\end{array}$ & $\begin{array}{c}\mathrm{NTM} \\
\mathrm{n}(\%)\end{array}$ & $\mathrm{n}$ & $\begin{array}{c}\text { Total } \\
(\mathrm{n})\end{array}$ \\
\hline Porto Velho & $296(87.3)$ & $43(12.7)$ & 882 & 1,221 \\
Ji-Paraná & $15(44.1)$ & $19(55.9)$ & 134 & 168 \\
Ariquemes & $15(71.4)$ & $6(28.6)$ & 62 & 83 \\
Others & $43(86)$ & $7(14)$ & 290 & 340 \\
\hline Total & 369 & 75 & 1,368 & 1,812 \\
\hline
\end{tabular}


TABLE II

Species identification of 75 isolates nontuberculous Mycobacterium according to municipalities between 2008-2010

\begin{tabular}{|c|c|c|c|c|c|}
\hline \multirow[b]{2}{*}{ Species } & \multicolumn{5}{|c|}{ Municipalities } \\
\hline & $\begin{array}{c}\text { Porto Velho } \\
\text { n }(\%)\end{array}$ & $\begin{array}{c}\text { Ji-Paraná } \\
\text { n }(\%)\end{array}$ & $\begin{array}{c}\text { Ariquemes } \\
\mathrm{n}(\%)\end{array}$ & $\begin{array}{l}\text { Others } \\
\text { n }(\%)\end{array}$ & $\begin{array}{c}\text { Total } \\
\mathrm{n}\end{array}$ \\
\hline Mycobacterium abscessus & $10(23.3)$ & $8(42)$ & $3(50)$ & $3(42.9)$ & 24 \\
\hline Mycobacterium avium & $10(23.3)$ & - & $2(33.3)$ & $1(14.3)$ & 13 \\
\hline Mycobacterium fortuitum & $4(9.3)$ & $4(21)$ & - & $1(14.3)$ & 9 \\
\hline Mycobacterium asiaticum & $1(2.3)$ & - & - & - & 1 \\
\hline Mycobacterium intracellulare & $1(2.3)$ & $3(15.8)$ & - & - & 4 \\
\hline Mycobacterium gordonae & $1(2.3)$ & $1(5.3)$ & - & - & 2 \\
\hline Mycobacterium szulgai & - & $1(5.3)$ & - & - & 1 \\
\hline Mycobacterium tusciae & $1(2.3)$ & - & - & - & 1 \\
\hline Mycobacterium phlei & - & $1(5.3)$ & - & - & 1 \\
\hline Mycobacterium simiae & $1(2.3)$ & - & - & - & 1 \\
\hline Mycobacterium novocastrense & $1(2.3)$ & - & - & - & 1 \\
\hline Mycobacterium gilvum & $2(4.6)$ & - & - & - & 2 \\
\hline Mycobacterium porcinum & $1(2.3)$ & - & - & - & 1 \\
\hline Mycobacterium holsaticum & $1(2.3)$ & - & - & - & 1 \\
\hline Not identified & $9(21)$ & $1(5.3)$ & $1(16.7)$ & $2(28.6)$ & 13 \\
\hline Total & 43 & 19 & 6 & 7 & 75 \\
\hline
\end{tabular}

TABLE III

Species identification of isolates which had concordant results by phenotypic and polymerase chain reaction-based restriction enzyme analysis- $h s p 65$ methods

\begin{tabular}{|c|c|c|c|c|}
\hline Samples & BSTEII & HAEIII & Profile compatible with & $\begin{array}{l}h s p 65 \text { gene sequencing } \\
(99.75 \% \text { ID) }\end{array}$ \\
\hline $157 / 08$ & $235 / 205$ & $160 / 145$ & Mycobacterium simiae type 2 & - \\
\hline $732 / 10$ & $235 / 205$ & $130 / 105$ & Mycobacterium avium type 1 & - \\
\hline 788/09 & $235 / 205$ & $110 / 105$ & Mycobacterium asiaticum type 1 & - \\
\hline $1161 / 08$ & $235 / 205$ & $130 / 105$ & M. avium type 1 & - \\
\hline $1493 / 08$ & $235 / 205$ & $200 / 70 / 60 / 55$ & Mycobacterium abscessus type 2 & - \\
\hline $61 / 09$ & $235 / 115 / 85$ & $150 / 130 / 70$ & Mycobacterium tuberculosis complex & - \\
\hline $198 / 08$ & $235 / 205$ & $145 / 130$ & M. avium type $3^{a}$ & $\begin{array}{c}\text { M. avium } \\
\text { ATCC25291_GQ153289.1 }\end{array}$ \\
\hline $212 / 10$ & 440 & $140 / 60 / 55$ & Mycobacterium novocastrense type 1 & $\begin{array}{c}\text { M. novocastrense } \\
\text { CIP105546_AF547862.1 }\end{array}$ \\
\hline $708 / 10$ & $235 / 115 / 85$ & $150 / 130 / 70$ & M. tuberculosis complex & - \\
\hline $725 / 10$ & 440 & $130 / 105 / 100$ & Mycobacterium szulgai type 1 & - \\
\hline $826 / 10$ & 440 & $140 / 100 / 60$ & Mycobacterium holsaticum type 1 & - \\
\hline $904 / 10$ & $235 / 205$ & $200 / 70 / 60 / 55$ & M. abscessus type 2 & - \\
\hline $1110 / 09$ & $235 / 115 / 85$ & $150 / 130 / 70$ & M. tuberculosis complex & - \\
\hline $1254 / 09$ & $235 / 115 / 85$ & $150 / 130 / 70$ & M. tuberculosis complex & - \\
\hline $188 / 08$ & 440 & $175 / 90$ & Mycobacterium tusciae & - \\
\hline $1603 / 08$ & 440 & $180 / 145$ & Mycobacterium gilvum & - \\
\hline 022/09 & $235 / 205$ & $140 / 120 / 100$ & $\mathrm{New}^{b}$ & $\begin{array}{c}\text { Mycobacterium boenickei } \\
\text { CIP } 107829\end{array}$ \\
\hline
\end{tabular}

a: seven species with the same pattern: Mycobacterium intracellulare type 3, M. avium s. avium type 3, Mycobacterium saskatchewanense type 1, Mycobacterium seoulense type 1, M. simiae type 6, Mycobacterium intermedium type 1, Mycobacterium interjectum type $1 ; b$ : three species with the same score: Mycobacterium porcinum type 1, Mycobacterium peregrinum type 2 and Mycobacterium septicum type 1. 


\section{DISCUSSION}

Unlike TB, notification of NTM-induced pulmonary disease is voluntary in Brazil and not confirmed by official records (Ueki et al. 2005, Pedro et al. 2008). In the present study, $16.9 \%$ of the Mycobacterium isolates were NTM and from our data, we calculated the incidence of NTM in 2010 as 3.9/100,000 and 10.8/100,000 in Porto Velho and Ji-Paraná, respectively, which is higher than the incidence of NTM-induced pulmonary disease reported in other studies conducted in Brazil. In an earlier study, Costa (2009) identified 51 NTM isolates positive for AFB in respiratory samples from 742 individuals $(6.9 \%)$ diagnosed for pulmonary TB in the state of Pará (PA), in North Brazil, between 2004-2007. In another study performed in the same state over a 12-year period, $8 \%$ of TB cases were found to be caused by NTM (Costa 2012). Although our study was also performed in the northern part of Brazil, we found that the NTM frequency was more than twice this value. Based on the analysis of samples from various regions of Brazil between 1994-1999, Barreto and Campos (2000) reported that the majority of positive cultures (433, corresponding with $73.3 \%$ ) for NTM were from pulmonary secretions.

The most frequently isolated NTM species in our samples were M. abscessus (32\%), M. avium (17.3\%) and M. fortuitum (12\%). The species Mycobacterium szulgai, $M$. novocastrense, Mycobacterium porcinum, Mycobacterium holsaticum, Mycobacterium asiaticum, Mycobacterium phlei and Mycobacterium simiae were isolated only in Porto Velho and were not the same species found at a higher frequency in other states. In PA, the most frequent species were Mycobacterium massiliense (17.6\%), M. avium (9.8\%), Mycobacterium colombiense (9.8\%), Mycobacterium intracellulare $(9.8 \%)$ and M. fortutuim (7.8\%) Costa (2009). Generally, however, the most frequent species of NTM associated with lung disease in Brazil are Mycobacterium kansasii and M. avium, while species such as Mycobacterium xenopi, Mycobacterium malmoense, Mycobacterium lentiflavum, M. abscessus and M. szulgai are isolated occasionally (Barreto \& Campos 2000, Chimara et al. 2008). However, most of the studies on the prevalence of NTM species have been conducted with residents of São Paulo, the country's largest metropolis. Analysis of pulmonary and extrapulmonary material sampled between 1991-1997 has established $M$. avium complex (MAC) $(64.9 \%)$ as the most frequent species, followed by $M$. kansasii $(12.2 \%)$ and Mycobacterium gordonae (10.2\%), although M. kansasii was the most frequently isolated species from the lung (83\%) (Ueki et al. 2005). Pedro et al. (2008) evaluated 317 strains of NTM and identified $182(57.4 \%)$ as MAC, $33(10.4 \%)$ as $M$. gordonae, $25(7.9 \%)$ as $M$. fortuitum, eight $(2.5 \%)$ as $M$. chelonae, seven (2.2\%) as M. kansasii and less prevalent species in 54 (17\%) samples. Zamarioli et al. (2008) recently reported $M$. kansasii as the most prevalent NTM species (33.6\%), higher than the MAC (17\%) and M. fortuitum (13.4\%). In the United States of America (USA), the most frequent NTM species associated with disease are MAC and other fast-growers, such as M. abscessus, M. chelonae and M. fortuitum (Cassidy et al. 2009). In Asia, rapidly growing mycobacteria were found to cause $14 \%$ of pulmonary NTM infections, but in India, Taiwan and South Korea this frequency was found to be $>30 \%$ (Simons et al. 2011). In England, where the frequency of NTM occurrence increased from $0.9 / 100,000$ people in 1995 to $2.9 / 100,000$ in 2006 , the most commonly reported species was $M$. avium-intracellulare, which accounted for $43 \%$ of NTM cases, followed by M. malmoense (14\%) and M. kansasii (13\%). $M$. gordonae was reported to have the biggest percentage increase over the study period (Moore et al. 2010).

The rarely encountered $M$. novocastrense was identified by molecular methods and to the best of our knowledege, this is the first description of this species in Brazil. However, our sequencing method was also unable to identify of a number of isolates that could not be identified by PRA and this is currently being investigated further. In Table III, the PRA pattern of the strain characterised as $M$. boenickei was not as reported previously (PRASITE - app.chuv.ch/prasite/index.html), but this could be a new genetic variant and second PRA pattern of this less-frequently described species.

The fact that 12 of the 14 NTM species observed in the present study were isolated in Porto Velho is most likely because $67.4 \%$ of the samples were from that region. The difference in the NTM species predominating in this region (more $M$. fortuitum and $M$. avium compared with M. abscessus) could be due to its geographical extent, including different biotopes and the larger population. In the study conducted in PA, Costa (2009) identified 12 different NTM species in respiratory specimens from 20042007. In another study in the state of São Paulo (SP), in the Santos region, Zamarioli (2008) found 13 NTM species in 194 isolates sampled between 2000-2005.

The high prevalence of $M$. abscessus confirms that this species is more frequently associated with pulmonary disease than other NTM species and this could be due to its abundance in the environment, high pathogenicity and resistance to drugs used for TB treatment. Although M. abscessus is rarely isolated from soil or water, there is general agreement that it is widely present in such environments (Macheras et al. 2011, Verregghen et al. 2012). This species is especially problematic due to its resistance to many antibiotics (Griffith et al. 2007) and when identified in the respiratory tract, it is usually associated with significant pulmonary disease (BrownElliott \& Wallace 2002).

A higher relative frequency of NTM isolates was observed in Ji-Paraná in 2010 due to a frequency increase during the three-year period from 2008-2010 that was not observed in other regions of the state (data not shown). We currently have no explanation for this relatively higher NTM frequency in the region. This observation is also striking because only $4.3 \%$ of the cultures from this region could be analysed due to operational problems and, as demonstrated in the Table II, $42 \%$ of the cases had M. abscessus.

The fact that we received a second sample from only half of the cases demonstrates the difficulty of the health program in following up with patients. Other studies have 
also reported similar constraints. A study performed in Santos (SP) reported that $80.8 \%$ of subjects had only one positive culture test (Zamarioli et al. 2008) and this is characteristic of developing countries in which limited resources often do not allow even culture isolation as a diagnostic option (Gebre et al. 1995). However, a study in the USA that observed an increase in NTM also reported problems, such as the inability to examine clinical records of patients and distinguish temporary illness from colonisation (Griffith et al. 2007). Given that $90.5 \%$ cases from which we obtained a second sample tested positive for NTM, it is very likely that this also is the case for subjects in which a second sample was unavailable. Therefore, the disease-causing frequency of NTM species in RO could be much higher than that deduced from our present data.

Among the 19 individuals who had at least two NTM-positive samples, two showed the presence of other species. The first case was an HIV-positive patient with one sample containing $M$. simiae and the second containing $M$. avium. The other case was an HIV-negative patient who presented three different NTM species in two samples. M. fortuitum was present in both samples, with M. szulgai in one case and M. gordonae in the other. We also observed three cases of NTM coinfection with $M$. tuberculosis, but the actual frequency of this phenomenon is unknown because all of the samples were investigated for the presence of the latter species. It has been shown that coinfection of NTM and $M$. $t u$ berculosis is not infrequent but rarely diagnosed (Gopinath \& Singh 2009).

Empirical use of fluoroquinolones and other antitubercular drugs in relatively less-efficient health care settings creates a false impression of a low incidence of nontubercular cases (Gopinath \& Singh 2010). However, we observed a high frequency of NTM-causing pulmonary disease cases in RO. Because many NTM species are resistant to several antibiotics used for TB treatment and treatment scheme depends on the NTM species involved, a delay in the identification of NTM species and delays in distinguishing between MTBC and NTM can lead to inadequate treatment. Therefore, we suggest the implementation of rapid and precise methods for NTM species identification in RO.

\section{ACKNOWLEDGEMENTS}

To Adalberto Rezende Santos, Fiocruz/RJ, Francisco Ivan Braga Faig and Maria Helena Morais Dias, CEMETRON/ RO, Raimunda Nonata Feitosa Rodrigues and Maria Luzinete Lopes Ribeiro, LACEN/RO, Ana Lúcia Teixeira, UBS/JiParaná/RO and Nilda de Oliveira Barros, AGEVISA/RO.

\section{REFERENCES}

Barreto AMW, Campos CED 2000. Micobactérias não-tuberculosas no Brasil. Bol Pneumol Sanit 8: 23-32.

Brachaman PS, Abrutyn E 2009. Bacterial infections of humans. Epidemiology and control, 4th ed, Springer, New York, 959 pp.

Brown-Elliott BA, Wallace Jr RJ 2002. Clinical and taxonomic status of pathogenic nonpigmented or late-pigmenting rapidly growing mycobacteria. Clin Microbiol Rev 15: 716-746.
Cassidy PM, Hedberg K, Saulson A, McNelly E, Winthrop KL 2009. Nontuberculous mycobacterial disease prevalence and risk factors: a changing epidemiology. Clin Infect Dis 49: e124-129.

Chimara E, Ferrazoli L, Leão SC 2008. Reliable identification of mycobacterial species by PCR-restriction enzyme analysis (PRA)-hsp65 in a reference laboratory and elaboration of a sequence-based extended algorithm of PRA-hsp65 patterns. BMC Microbiol 8: 48.

Costa ARF 2009. Diversidade genética das espécies de micobactérias não tuberculosas identificadas em laboratório de referência para o diagnóstico da tuberculose na Região Norte, MsD, Universidade Federal do Pará, Belém, 103 pp.

Costa ARF 2012. Pulmonary nontuberculosis mycobacterial infections in the state of Pará, an endemic region for tuberculosis in North of Brazil. In Pulmonary infection, INTECH Open Acess Publisher, Rijeka, p. 390-398.

Dai J, Chen Y, Dean S, Morris JG, Salfinger M, Johnson JA 2011. Multiple-genome comparison reveals new loci for Mycobacterium species identification. J Clin Microbiol 49: 144-153.

Gebre N, Karlsson U, Jönsson G, Macaden R, Wolde A, Assefa A, Miörner H 1995. Improved microscopical diagnosis of pulmonary tuberculosis in developing countries. Trans $R$ Soc Trop Med Hyg 89: 191-193.

Gopinath K, Singh S 2009. Multiplex PCR assay for simultaneous detection and differentiation of Mycobacterium tuberculosis, Mycobacterium avium complexes and other mycobacterial species directly from clinical specimens. J Appl Microbiol 107: 425-435.

Gopinath K, Singh S 2010. Non-tuberculous mycobacteria in TB-endemic countries: are we neglecting the danger? PLoS Negl Trop Dis 4: e615.

Griffith DE, Aksamit T, Brown-Elliott BA, Catanzaro A, Daley C, Gordin F, Holland SM, Horsburgh R, Huitt G, Iademarco MF, Iseman M, Olivier K, Ruoss S, von Reyn CF, Wallace Jr RJ, Winthrop K 2007. An official ATS/IDSA statement: diagnosis, treatment and prevention of nontuberculous mycobacterial diseases. Am J Respir Crit Care Med 175: 367-416.

Katoch VM 2004. Infections due to non-tuberculous mycobacteria (NTM). Indian J Med Res 120: 290-304.

Macheras E, Roux AL, Bastian S, Leão SC, Palaci M, Sivadon-Tardy V, Gutierrez C, Richter E, Rüsch-Gerdes S, Pfyffer G, Bodmer T, Cambau E, Gaillard JL, Heym B 2011. Multilocus sequence analysis and rpoB sequencing of Mycobacterium abscessus (sensu lato) strains. J Clin Microbiol 49: 491-499.

McNabb A, Eisler D, Adie K, Amos M, Rodrigues M, Stephens G, Black WA, Isaac-Renton J 2004. Assessment of partial sequencing of the 65-kilodalton heat shock protein gene (hsp65) for routine identification of Mycobacterium species isolated from clinical sources. J Clin Microbiol 42: 3000-3011.

Moore JE, Kruijshaar ME, Ormerod LP, Drobniewski F, Abubakar I 2010. Increasing reports of non-tuberculous mycobacteria in England, Wales and Northern Ireland, 1995-2006. BMC Public Health 10: 612.

MS - Ministério da Saúde 2005. Manual de bacteriologia da tuberculose, 3rd ed., MS, Brasília DF, 240 pp.

MS - Ministério da Saúde 2008. Manual nacional de vigilância laboratorial da tuberculose e outras micobactérias, MS/Secretaria de Vigilância em Saúde/Departamento de Vigilância Epidemiológica, Brasília DF, 436 pp.

Pedro H da SP, Pereira MIF, Goloni M do RA, Ueki SYM, Chimara E 2008. Nontuberculous mycobacteria isolated in São José do 
Rio Preto, Brazil between 1996 and 2005. J Bras Pneumol 34: 950-955.

Simons S, Ingen JV, Soolingen DV 2011. Nontuberculous mycobacteria in respiratory tract infections, Eastern Asia. Emerg Infect Dis 17: 343-349.

Telenti A, Marchesi F, Balz M, Bally F, Böttger EC, Bodmer T 1993. Rapid identification of mycobacteria to the species level by polymerase chain reaction and restriction enzyme analysis. $J$ Clin Microbiol 31: 175-178.

Ueki SYM, Martins MC, Telles MA, Virgilio MC, Giampaglia CMS, Chimara E, Ferrazoli L 2005. Micobactérias não-tuberculosas: diversidade das espécies no estado de São Paulo. J Bras Patol Med Lab 41: 1-8.

Verregghen M, Heijerman HG, Reijers M, van Ingen J, van der Ent CK 2012. Risk factors for Mycobacterium abscessus infection in cystic fibrosis patients; a case-control study. J Cyst Fibros 11: 340-343.

Wallace Jr RJ, Brown BA, Griffith DE 1998. Nosocomial outbreaks/ pseudo-outbreaks caused by nontuberculous mycobacteria. Annu Rev Microbiol 52: 453-490.

Zamarioli LA, Coelho AGV, Pereira CM, Nascimento ACC, Ueki SYM, Chimara E 2008. Descriptive study of the frequency of nontuberculous mycobacteria in the Baixada Santista region of the state of São Paulo, Brazil. J Bras Pneumol 34: 590-594. 